\title{
Odin: Um modelo de coleta adaptativa de sinais vitais
}

\author{
Jorge Arthur Schneider Aranda \\ Universidade do Vale do Rio dos Sinos \\ São Leopoldo, RS, Brasil \\ jsaranda@unisinos.br
}

\author{
Juliano Varella de Carvalho \\ Universidade Feevale \\ Novo Hamburgo, RS, Brasil \\ julianovc@feevale.br
}

\author{
Jorge Luis Victória Barbosa \\ Universidade do Vale do Rio dos Sinos \\ São Leopoldo, RS, Brasil \\ jbarbosa@unisinos.br
}

\begin{abstract}
Wearable devices emerged from the advancement of communication technology and the miniaturization of electronic components. These devices periodically monitor the user's vital signs and generally have low battery life. This thesis proposes Odin, a model for optimized collection of vital signs based on adaptive strategies. The comparison with related works indicates that Odin is the only proposal that presents an adaptive collection of vital signs, which allows the optimized generation of context history. The research validation is divided in three groups. In the first group, simulations were performed in different scenarios, through requisition control, an increase of $114 \%$ in battery life was obtained in the adaptive scenario compared to a collection without adaptivity. In the second group, the Odin model was applied to a real physiological database and allowed for a reduction in the number of requests due to the adaptability of the collection. This reduction optimized battery consumption by $66 \%$ compared to a collection without adaptability. Finally, in the third group, the model was applied in collecting data through an Arduino and also a cardiac monitoring strap available on the market (Polar H7). During this third part of the evaluation, savings of $18 \%$ were observed in the power consumption of the mobile devices involved in the data collection.
\end{abstract}

\section{KEYWORDS}

E-health, Multiagents, Ubiquitous Computing, Adaptive Systems

\section{INTRODUÇÃO}

Este artigo apresenta um resumo dos resultados obtidos em uma dissertação de mestrado conduzida no Programa de Pós-graduação em Computação Aplicada (PPGCA) na Universidade do Vale do Rio dos Sinos (UNISINOS). O trabalho foi realizado em 24 meses, tendo a banca ocorrido no dia 18/04/2020. A dissertação proporcionou a publicação de 5 artigos em eventos e periódicos, dentre os quais destacam-se uma revisão bibliográfica [2] e uma descrição do modelo computacional proposto [1]. Ambos os artigos foram publicados no periódico fournal of Ambient Intelligence and Humanized Computing, editado pela Elsevier. Além disso, três softwares derivados da dissertação foram registrados no INPI sob os números BR512021001281-0, BR512021001279-8 e BR512021001280-1.

Nota-se que nos últimos anos, com o envelhecimento da população mundial [17], vem ocorrendo uma demanda por cuidados médicos de qualidade em qualquer ambiente. As soluções de saúde móvel desempenham um papel importante na coleta de dados para

In: III Concurso de Teses e Dissertações (CTD 2021), Minas Gerais, Brasil. Anais Estendidos do Simpósio Brasileiro de Sistemas Multimídia e Web (WebMedia). Porto Alegre: Sociedade Brasileira de Computação, 2021.

(c) 2021 SBC - Sociedade Brasileira de Computação.

ISSN 2596-1683 monitorar o estado do paciente e podem reduzir o custo dos serviços médicos. Embora esforços venham sendo dedicados a melhorar a arquitetura e a conectividade entre dispositivos [9], menos atenção foi dedicada ao desenvolvimento de técnicas analíticas para avaliar o estado de saúde atual das pessoas.

A computação ubíqua pode ser considerada uma extensão da computação móvel, pois ela herda parte de suas características, como transparência e sensibilidade ao contexto [16]. Estes contextos são informações que podem estar relacionadas com pessoas (indivíduos, grupos), tais como sinais vitais (batimentos cardíacos, temperatura corporal) ou relacionados com coisas (dispositivos vestíveis, recursos computacionais). Os contextos podem possuir atributos, como uma identidade (identificação única), status e tempo (para definir a ordenação que os eventos ocorrem) [5, 16].

Conforme Wagner et al. [19], a adaptação em contextos deve se basear nos atributos disponibilizados de forma a compreender a situação do usuário. Porém, apenas o contexto atual (instantâneo) pode não fornecer a totalidade de informações necessárias, por isso é importante acompanhar os históricos de contextos [3] onde a entidade está inserida.

Este artigo descreve o modelo Odin que realiza análises em tempo real de dados fisiológicos para monitorar as condições de saúde dos usuários, atuando sobre os parâmetros de coleta de sinais vitais. Os históricos de contextos fisiológicos são analisados, permitindo que a coleta se adapte e seja otimizada. O texto está dividido em cinco seções. A segunda e a terceira abordam os trabalhos relacionados e o modelo proposto, respectivamente. A seção quatro apresenta os aspectos de avaliação. Por fim, a seção cinco aborda as conclusões.

\section{TRABALHOS RELACIONADOS}

Este trabalho utilizou um mapeamento sistemático como metodologia para revisão da literatura [2]. O mapeamento identificou 10 artigos de coleta, transmissão e análise de sinais vitais, os quais foram considerados como trabalhos relacionados.

O mapeamento foi organizado em três etapas: (1) identificação de questões de pesquisa; (2) elaboração do processo de busca; (3) definição dos critérios para a filtragem dos resultados. A comparação com trabalhos relacionados indica que Odin é a única proposta que apresenta uma coleta adaptativa de sinais vitais, o que permite a geração otimizada de históricos de contextos.

\section{O MODELO ODIN}

A Figura 1 mostra a arquitetura do modelo Odin baseada no padrão Technical Architecture Module (TAM) da SAP [15]. O Odin é composto por quatro módulos: Agentes, Backend, Frontend e Database. O módulo de Agentes está relacionado a interação com os dispositivos de coleta de sinais vitais. O módulo de Backend consiste no 
Controller de regras, de notificações e de sinais vitais. Estes Controllers permitem o acesso aos históricos de contextos armazenados no módulo Database. O Frontend possui as Views de regras, de sinais vitais e de alerta. Estas Views são responsáveis por exibir as informações aos usuários. Por fim, o módulo Database armazena os históricos de contextos dos usuários, assim como suas informações pessoais e regras, que são representadas através de um fluxograma.

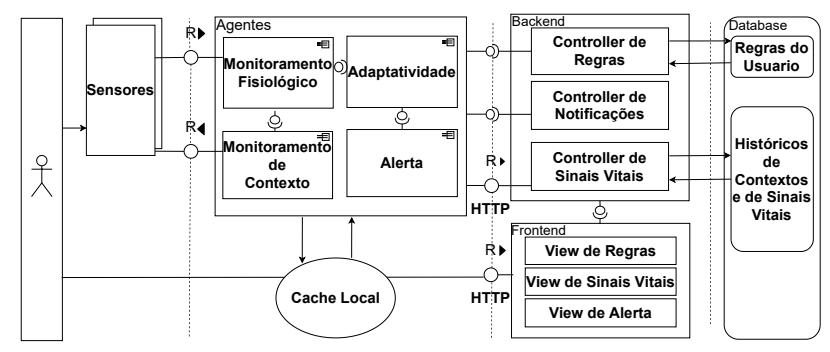

Figura 1: Arquitetura do Modelo Odin

\subsection{Organização MultiAgentes do Odin}

Os agentes do Odin foram modelados usando a metodologia Prometheus $[13,14]$. Os cinco agentes utilizados são os agentes de Monitoramento Fisiológico, Monitoramento de estado, Sincronização, Alerta e Adaptabilidade. A Figura 2 mostra a distribuição multiagentes proposta.

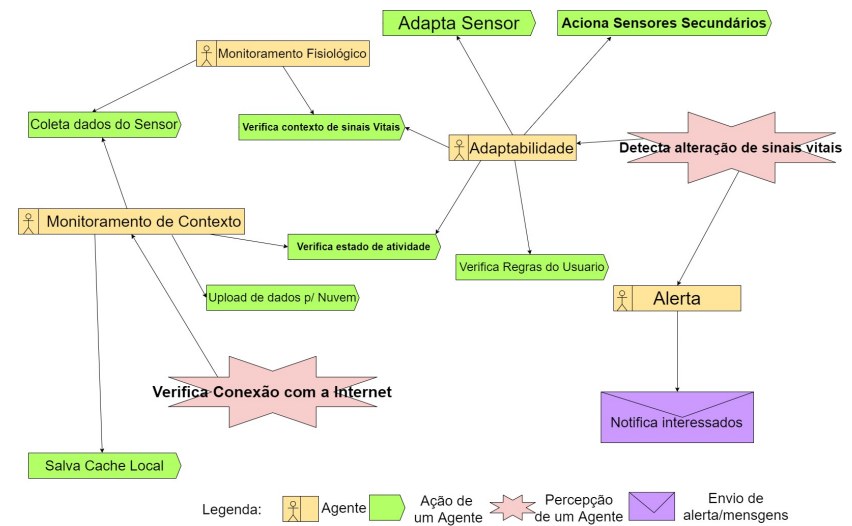

Figura 2: Distribuição Multiagentes

O agente de Monitoramento Fisiológico é responsável pela interface de comunicação com os sensores de sinais vitais, disponibilizando os dados coletados para os demais agentes. $\mathrm{O}$ agente de Monitoramento de Contexto é responsável por coletar informações de sensores como acelerômetro e giroscópio, podendo estes estarem no próprio celular ou no dispositivo vestível. O agente de Alerta exibe notificações ao usuário de acordo com as adaptações que estão sendo efetuadas pelo agente de Adaptabilidade.

$\mathrm{O}$ agente de Adaptabilidade efetua dois tipos de adaptações. $\mathrm{O}$ primeiro tipo ocorre através da alteração do parâmetro de tempo de coleta, denominado Adaptação no Tempo de Espera (ATE). Se os sinais vitais saírem dos valores considerados regulares, o agente altera a periodicidade de coleta do sensor para uma maior frequência. Se estes valores continuarem a progredir em direção oposta aos valores regulares, o tempo de espera entre coletas é diminuído ainda mais. Quanto mais próximo dos valores regulares de sinais vitais a coleta estiver, maior será o tempo de espera entre coletas. Com os valores dentro do limiar de valores regulares a coleta atinge o tempo de espera máximo. Todas estas alterações são efetuadas automaticamente pelo agente de Adaptabilidade. O segundo tipo de adaptação consiste em acionar um ou mais sensores secundários que porventura estejam em estado de pausa. Esta adaptação tem o nome de Adaptação de Sensores em Pausa (ASP). Se o usuário possuir um dispositivo vestível com mais de um tipo de sensor de sinais vitais, um ou mais destes sensores podem ser definidos como Sensor Primário (SP) e o restante como Sensor(es) Secundário(s) (SS). Isso permite que um SS apenas seja acionado quando um SP estiver fora do limiar de sinais vitais regulares. A ASP irá iniciar ou pausar um SS de acordo com o contexto fisiológico do usuário de maneira automatizada.

\subsection{Regras de Adaptação}

As regras são representadas através de um fluxograma baseado em evento, condição e ação. O evento se baseia na coleta de sinais vitais, a verificação da condição é feita em três etapas e ao final da determinação da condição, que seria a adaptação da coleta. A primeira etapa verifica qual a condição de estado do usuário (em repouso ou em movimento), a segunda etapa, verifica o perfil do usuário, identificando se ele é um adulto, uma criança ou um idoso. Com base nessas informações é possível examinar a terceira etapa, de forma a utilizar o padrão correto de condições fisiológicas do usuário. A Figura 3 mostra o fluxograma das regras de adaptação.

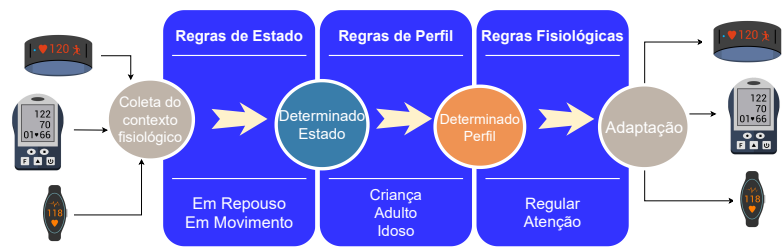

Figura 3: Regras de Adaptação

Uma vez determinada a condição fisiológica do usuário, a mesma pode ser classificada em estado regular ou estado de alerta. A padronização dos tipos e valores de sinais vitais seguem as definições propostas pelo Ministério da Saúde [12].

\subsection{Fuzzyficação das regras}

A utilização de agentes aliada ao mapeamento de possíveis condições fisiológicas em forma de regras, permite efetuar uma inferência da condição de saúde do usuário. Para verificar o nível da condição do usuário utilizando lógica fuzzy, se faz necessário um cálculo da diferença do valor de sinais vitais aferido em relação ao limiar normal daquele tipo de sinal vital, conforme a fórmula descrita pela Equação 1. Estes limiares podem variar de acordo com o perfil do usuário (idade, gênero) [18]. 


$$
\text { sinalVitalFuzzyficado }=\frac{\text { sinalVitalAferido }- \text { limiarSinalVital }}{\text { limiarSinalVital }}
$$

$\mathrm{O}$ valor obtido pode ser um número real de 0 a 1 , onde 0 seria correspondente a um valor exatamente igual ao limiar e 1 um valor crítico, $100 \%$ distante do limiar. Para o cálculo e inferência das regras, mais informações podem ser relevantes, como o estado de atividade do usuário ou o seu grau de sedentarismo. Estudos [7, 8] alertam para a importância do exercício no impacto da saúde mental. Dessa forma informações sobre o grau de sedentarismo do usuário se tornam informações contextuais importantes. Porém essas informações são bastante subjetivas e para correlacionar com valores de sinais vitais o ideal seria exprimir um valor numérico para estas condições.

Para utilizar lógica fuzzy, a fim de determinar o nível de atividade de um usuário, torna-se necessário calcular a diferença em milissegundos de uma incidência de movimento para outra. Esta incidência pode ser obtida através de um acelerômetro presente em dispositivos vestíveis ou smartphones. Por exemplo, um usuário em uma caminhada moderada possuirá um valor em milissegundos alto, enquanto um usuário correndo, possuirá um número baixo devido à maior intensidade da atividade. Um exemplo de uma incidência de atividade neste caso poderia ser a diferença de tempo em milissegundos de dois passos dados por um usuário. Para estimar quantas incidências desta atividade são feitas por minuto é necessário encontrar a razão de 60000 (1 minuto em milissegundos) pela diferença de tempo entre os passos. Através do número de passos por minuto é possível definir a intensidade $[4,6,11]$ da atividade através de um limiar, onde 0 seria referente a um usuário parado e 1 seria o usuário em altíssima intensidade, conforme fórmula de cálculo da atividade descrita na Equação 2.

$$
\text { atividadeFuzzyficada }=\frac{60000 / \text { diferencaEntrePassos }}{\text { limiarAtividade }}
$$

Um usuário com batimentos cardíacos acima de 100 pode ser considerado taquicárdico, porém se o mesmo está em movimento, a intensidade da atividade é considerada, através de um cálculo de média ponderada entre os valores fuzzy, evitando que a inferência da regra gere um alerta ou faça uma adaptação quando não exista uma situação fora dos padrões normais de sinais vitais.

\section{ASPECTOS DE AVALIAÇÃO}

Foram utilizados cinco tipos de avaliações organizados em três grupos. Dentro do primeiro grupo foram desenvolvidos dois cenários onde a geração de dados se obteve através de simulação. No segundo grupo de avaliações, o modelo foi aplicado em uma base de dados fisiológicos reais. Utilizando estes dados como se estivessem sendo coletados de um usuário observou-se a redução da quantidade de requisições devido à adaptabilidade. No terceiro grupo de avaliação, foram efetuados mais dois experimentos utilizando um protótipo de coletas de sinais vitais, assim como uma cinta de monitoramento cardíaco disponível no mercado. O protótipo foi desenvolvido em Arduino e a cinta de monitoramento cardíaco utilizada foi a Polar modelo H7. Em ambos os casos também foi utilizado uma aplicação de controle, onde a adaptação de requisições não era efetuada. Com a aplicação de controle foi possível comparar as diferenças entre uma coleta adaptativa e uma coleta regular de sinais vitais.

- Grupo 1-Simulação - Cenário 1: Simulação de cenário de um usuário que busca apenas mapear seu nível de estresse. O objetivo desta avaliação é monitorar um usuário em seu cotidiano, sem a aplicação de um contexto específico.

- Grupo 1-Simulação - Cenário 2: Cenário em um contexto de usuário que pratica esportes. Essa simulação tem como objetivo avaliar o uso do modelo em um contexto de um atleta amador.

- Grupo 2-Base de Dados: Utilização de um dataset de sinais vitais, estes dados foram coletados em ambiente hospitalar de pacientes na UTI [10]. Esta avaliação foi desenvolvida para verificar o comportamento do modelo ao utilizar dados fisiológicos reais.

- Grupo 3-Coleta de sinais vitais - Arduino: Coleta através de um protótipo desenvolvido em Arduino. Essa avaliação tem como objetivo verificar a viabilidade do modelo proposto e o seu funcionamento em um ambiente real.

- Grupo 3-Coleta de sinais vitais - Polar H7: Coleta através de uma cinta de monitoramento cardíaco. O objetivo desta avaliação é de verificar a coleta e o funcionamento da aplicação em um dispositivo vestível disponível no mercado.

\subsection{Resultado dos Cenários (Grupo 1)}

Através da aplicação dos cenários foi possível verificar dois resultados. No cenário 1, o médico pode analisar por meio dos históricos de contextos, quanto tempo o usuário ficou em uma possivel condição de risco. No cenário 2, foi possível identificar quanto tempo o usuário ficou com risco de hipotermia, a temperatura neste cenário foi um SS e somente foi acionado quando o SP (batimentos cardíacos) saia dos padrões regulares. Por consequência da adaptação a autonomia de bateria dos dispositivos mencionados nos cenários 1 e 2 foi aumentada. No primeiro cenário houve um aumento de $114 \%$, já no segundo cenário foi encontrando um aumento de $164 \%$ de autonomia de bateria quando comparado à coleta de sinais vitais sem adaptação.

\subsection{Avaliação com dados de pacientes (Grupo 2)}

Os dados monitorados neste dataset consistem em batimentos cardíacos e pressão arterial. A utilização de dados reais pode sugerir uma alteração de sinais vitais mais próxima de uma situação real, permitindo uma melhor avaliação da adaptabilidade de dados fisiológicos. Para esta avaliação foram definidos alguns parâmetros. No dataset utilizado, o contexto fisiológico do usuário foi coletado durante 3 horas. O tempo de espera entre as coletas foi definido como 10 segundos para um valor abaixo de $20 \%$ de estresse e 1 segundo para uma condição acima disso. Também foi definido que o SP seria o de Variação de Frequência Cardíaca (VFC), enquanto os sensores de batimentos cardíacos e pressão arterial foram definidos como SS. Com a adaptação foram efetuados 3590 requests em comparação as 10761 requisições sem adaptação, uma redução de 66,64\%.

\subsection{Uso com dispositivos vestíveis (Grupo 3)}

Para a coleta de sinais vitais foi desenvolvido um protótipo de hardware utilizando Arduino uno. Em conjunto com o Arduino foram 
utilizados um shield bluetooth ESP32 e um sensor de frequência cardíaca. Também foi utilizado um suporte de pilhas AA para alimentar o dispositivo durante o seu uso. A utilização do protótipo se deu através de uma braçadeira de celular e utilizado na altura do pulso. O sensor de frequência cardíaca foi preso ao dedo indicador com ajuda de uma fita. O suporte de pilhas e o shield bluetooth ficaram presos dentro da braçadeira. Este experimento utilizou 4 pilhas AA recarregáveis a fim de alimentar o protótipo. Os testes foram executados duas vezes utilizando o app com adaptação e mais duas vezes utilizando o aplicativo de controle. Fazendo uma média entre as duas coletas, verificou-se um aumento de cerca de $18 \%$ na autonomia de bateria do dispositivo. Por outro lado, o número de requisições foi $47,72 \%$ menor em comparação ao experimento sem adaptação. O segundo dispositivo usado nessa validação foi a polar H7, que é uma cinta cardíaca utilizada dentro da camisa do usuário. Para a avaliação através deste dispositivo vestível, foi possível apenas observar a autonomia de bateria do smartphone utilizado, uma vez que o vestível não disponibiliza acesso ao seu total de bateria. Novamente foram efetuados dois testes utilizando a adaptação e mais dois testes sem adaptação através da aplicação de controle. A duração dos testes ficou limitada ao tempo de autonomia de bateria do smartphone. Analisando as coletas com a cinta Polar, verificou-se um aumento de cerca de $19 \%$ na autonomia de bateria do dispositivo. Por outro lado, o número de requisições foi 44,13\% menor em comparação ao experimento sem adaptação.

\section{CONCLUSÃO}

Este artigo descreveu um modelo denominado Odin para coleta adaptativa de sinais vitais. Para a criação do modelo, foram levados em conta os trabalhos relacionados, de forma a encontrar funcionalidades ainda não oferecidas como a coleta de sinais vitais que se adapta ao usuário. Ao realizar a avaliação foi possível identificar quanto tempo o usuário fica em uma possível situação de risco através da análise dos históricos de contextos de sinais vitais, uma vez que este histórico é alimentado pela coleta adaptativa de dados fisiológicos.

Devido a dificuldade de interoperabilidade de dispositivos móveis, este trabalho possui uma limitação. Apenas alguns dispositivos vestíveis comerciais permitem a alteração de parâmetros de sinais vitais, estando o Odin restrito a aplicar a solução proposta apenas a estes dispositivos. Futuras avaliações incluirão testes de acurácia das medições de sinais vitais, assim como testes comparativos de economia de energia, de forma a verificar se o processamento adicional necessário para a adaptação não é maior que a redução de energia obtida por meio da otimização das requisições.

\section{AGRADECIMENTOS}

Os autores agradecem à Fundação de Amparo à Pesquisa do Estado do Rio Grande do Sul (FAPERGS), à Coordenação de Aperfeiçoamento de Pessoal de Nível Superior - Brasil (CAPES) - Código de Financiamento 001, ao Conselho Nacional de Desenvolvimento Científico e Tecnológico (CNPq), à Universidade do Vale do Rio dos Sinos (Unisinos) e à Universidade Feevale pelo apoio ao desesenvolvimento deste trabalho.

\section{REFERÊNCIAS}

[1] Jorge Arthur Schneider Aranda, Rodrigo Simon Bavaresco, Juliano Varella de Carvalho, Adenauer Corrêa Yamin, Mauricio Campelo Tavares, and Jorge Luis Victória Barbosa. 2021. A computational model for adaptive recording of vital signs through context histories. Fournal of Ambient Intelligence and Humanized Computing (March 2021). https://doi.org/10.1007/s12652-021-03126-8

[2] Jorge Arthur Schneider Aranda, Lucas Pfeiffer Salomão Dias, Jorge Luis Victória Barbosa, Juliano Varella de Carvalho, João Elison da Rosa Tavares, and Mauricio Campelo Tavares. 2019. Collection and analysis of physiological data in smart environments: a systematic mapping. Fournal of Ambient Intelligence and Humanized Computing (July 2019). https://doi.org/10.1007/s12652-019-01409-9

[3] Jorge Barbosa, João Tavares, Ismael Cardoso, Bruno Alves, and Bruno Martini. 2018. TrailCare: An indoor and outdoor Context-aware system to assist wheelchair users. International fournal of Human-Computer Studies 116 (2018), 1 - 14. https://doi.org/10.1016/j.ijhcs.2018.04.001

[4] David R. Bassett, Holly R. Wyatt, Helen Thompson, John C. Peters, and James O. Hill. 2010. Pedometer-Measured Physical Activity and Health Behaviors in U.S. Adults. Medicine \& Science in Sports \& Exercise 42, 10 (Oct. 2010), 1819-1825. https://doi.org/10.1249/mss.0b013e3181dc2e54

[5] Anind K Dey and Gregory D Abowd. 2001. A Conceptual Framework and a Toolkit for Supporting the Rapid Prototyping of Context-Aware Applications. Journal Human-Computer Interaction 16 (2001), 97-166.

[6] Barbara e. Ainsworth, william l. Haskell, stephen d. Herrmann, nathanael meckes, david r. Bassett, catrine tudor locke, jennifer l. Greer, jesse vezina, melicia c. Whitt-glover, and arthur s. Leon. 2011. 2011 Compendium of Physical Activities. Medicine \& Science in Sports \& Exercise 43, 8 (Aug. 2011), 1575-1581. https: $/ /$ doi.org/10.1249/mss.0b013e31821ece12

[7] William e. Kraus, carol e. Torgan, brian d. Duscha, jami norris, spencer a. Brown, frederick r. Cobb, connie w. Bales, brian h. Annex, gregory p. Samsa, joseph a. Houmard, and cris a. Slentz. 2001. Studies of a targeted risk reduction intervention through defined exercise (STRRIDE). Medicine \& Science in Sports \& Exercise 33, 10 (Oct. 2001), 1774-1784. https://doi.org/10.1097/00005768-200110000-00025

[8] Johanna L. Johnson, Cris A. Slentz, Leanna M. Ross, Kim M. Huffman, and William E. Kraus. 2019. Ten-Year Legacy Effects of Three Eight-Month Exercise Training Programs on Cardiometabolic Health Parameters. Frontiers in Physiology 10 (April 2019). https://doi.org/10.3389/fphys.2019.00452

[9] Val Jones, Aart van Halteren, Ing Widya, Nikolai Dokovsky, George Koprinkov, Richard Bults, Dimitri Konstantas, and Rainer Herzog. 2006. Mobihealth: Mobile Health Services Based on Body Area Networks. Springer US, Boston, MA, 219-236. https://doi.org/10.1007/0-387-26559-7_16

[10] David Liu, Matthias Görges, and Simon A Jenkins. 2011. University of Queensland Vital Signs Dataset: Development of an Accessible Repository of Anesthesia Patient Monitoring Data for Research. Anesthesia and analgesia 114 (12 2011), 584-9. https://doi.org/10.1213/ANE.0b013e318241f7c0

[11] Constance M. Mier and Yuri Feito. 2006. Metabolic Cost of Stride Rate, Resistance, and Combined Use of Arms and Legs on the Elliptical Trainer. Research Quarterly for Exercise and Sport 77, 4 (Dec. 2006), 507-513. https://doi.org/10.1080/02701367. 2006.10599385

[12] Ministério da Saúde. Secretaria de Gestão do Trabalho e da Educação na Saúde. 2003. Departamento de Gestão da Educação na Saúde. Projeto de Profissionalização dos Trabalhadores da área de Enfermagem (2003).

[13] Muaz Niazi and Amir Hussain. 2011. Agent-based computing from multi-agent systems to agent-based Models: a visual survey. Scientometrics 89 (11 2011), 479-499. https://doi.org/10.1007/s11192-011-0468-9

[14] Lin Padgham and Michael Winikoff. 2004. Developing Intelligent Agent Systems: A Practical Guide. John Wiley \& Sons, Inc., New York, NY, USA. https://doi.org/ $10.1002 / 0470861223$

[15] SAP. 2007. Standardized Technical Architecture Modeling, (2007).

[16] M. Satyanarayanan. 2001. Pervasive computing: vision and challenges. IEEE Personal Communications 8, 4 (Aug 2001), 10-17. https://doi.org/10.1109/98. 943998

[17] United Nations Department of Economic and Social Affairs. 2019. World Population Prospects 2019. https://population.un.org/wpp/Publications/Files/ WPP2019_Highlights.pdf

[18] Andreas Voss, Rico Schroeder, Andreas Heitmann, Annette Peters, and Siegfried Perz. 2015. Short-Term Heart Rate Variability-Influence of Gender and Age in Healthy Subjects. PLOS ONE 10, 3 (March 2015), e0118308. https://doi.org/10. 1371/journal.pone. 0118308

[19] André Wagner, Jorge Luis Victória Barbosa, and Débora Nice Ferrari Barbosa. 2014. A model for profile management applied to ubiquitous learning environments. Expert Systems with Applications 41, 4, Part 2 (2014), 2023 - 2034. https://doi.org/10.1016/j.eswa.2013.08.098 